\title{
Electrical Energy Generated by Silicone Elastomers Filled with Nanospring-Carbon- Nanotubes
}

Yun Jae Lee, $\dagger^{a, b}$ Philip Caspari, $\dagger^{c, d}$ Dorina M. Opris, ${ }^{* c}$ Frank A. Nüesch, ${ }^{c, d}$ Sora Ham, ${ }^{a, e}$ Jung-Hyuk Kim, ${ }^{a, b}$ Sung-Ryong Kim, ${ }^{f}$ Byeong-Kwon Ju, and Won Kook Choi*a,e

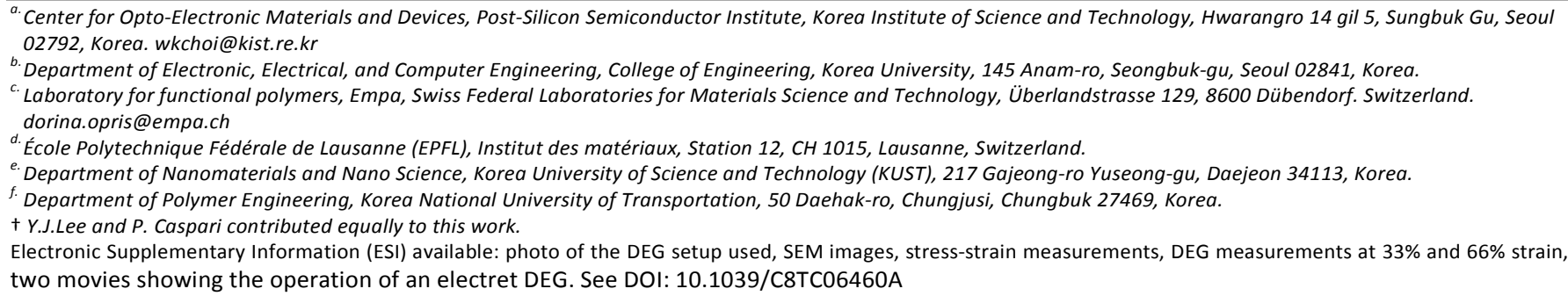

Dielectric elastomer generators (DEGs) are flexible capacitors that convert mechanical into electrical energy. They rely on an external voltage source to charge the capacitor during each operation cycle. An alternative to this is an autonomous DEG which operates with an electret as integrated voltage source. To increase the amount of energy generated per cycle, elastomers with increased permittivity, low viscoelastic losses and high strain at break are of advantage. Here, we report the synthesis of elastic materials with increased permittivity by blending different silicone matrices with nanospring carbonnanotubes (NS-CNTs) particles and their performance as dielectric in electret DEGs was evaluated. The best material developed has a dielectric permittivity of $\varepsilon^{\prime}=4.6$, a mechanical loss factor of 0.03 and a strain at break of $270 \%$. The output voltage of the DEG constructed using this composite increases from 8.8 $\mathrm{V}$ to $14.5 \mathrm{~V}$, when the strain increases from $33 \%$ to $66 \%$, respectively. Additionally, the output voltage increases with the rise in permittivity, from $9.3 \mathrm{~V}$ for a regular polydimethylsiloxane elastomer $\left(\varepsilon^{\prime}=2.9\right)$ to 14.5 for the best composite $\left(\varepsilon^{\prime}=4.6\right)$.

\section{Introduction}

The search for new functional materials and devices that produce reliable energy from green sources has increased significantly in the last years. Nature offers a number of sources of unlimited energy such as ocean waves, wind, and flow energy from rivers. ${ }^{1}$ Dielectric elastomer generators (DEGs) are stretchable capacitors capable of converting mechanical into electrical energy. ${ }^{2-4}$ They consist of a thin elastic dielectric film, on whose surface two compliant electrodes are deposited. The DEG changes its capacitance during stretching. When the elastic capacitor is stretched and charged at a certain voltage, and then let relax by the elastic restoring forces, a change in voltage is observed. This allows converting mechanical energy into electrical energy. ${ }^{5-10}$ The maximal energy converted by DEG is limited by the dielectric breakdown, dielectric permittivity and mechanical properties such as viscoelastic losses and strain at break of the dielectric elastomer used. ${ }^{8,11,12}$ Several DEG prototypes that harvest energy from waves and rivers flow, ${ }^{13-16}$ or human body motion were developed and showed promising performance. ${ }^{17-19}$

Polyurethanes, ${ }^{7,20}$ natural rubber, ${ }^{21}$ polyacrylates, ${ }^{19,22-25}$ and PDMS elastomers ${ }^{14,26-30}$ are among the most intensely studied dielectric elastomers for DEG applications. PDMS dielectric elastomers show superior mechanical stability, ${ }^{31}$ chemical inertness and fast response speed $^{32}$ combined with excellent insulating properties. ${ }^{33,34}$ However, the permittivity of PDMS elastomers is about 3 , which is lower than that of polyurethane or polyacrylate elastomers. Chemical modification of polysiloxanes with polar groups and blending polysiloxanes with conductive fillers like Ag nanoparticles, polyaniline, polythiophene, and CNT have been used to increase the permittivity of polysiloxanes. For some of these materials it was predicted that the amount of energy harvested is increasing with the permittivity. ${ }^{35-37}$ Despite the abundant literature on high permittivity elastomers ${ }^{38-42}$ and composites, ${ }^{43-47}$ reports regarding the use of such materials in generators are rare. To the best of our knowledge only one report by Wang et al. exists, in which materials with increased permittivity were evaluated as dielectric for DEG. ${ }^{7}$ The limited literature on high permittivity elastomers for DEG applications may be due to the complex setup used for the energy harvesting cycle which poses safety issues, because of the high voltage and current involved. A solution to this may be the use of electret DEGs introduced by Sylvestre et al. ${ }^{27,48-50}$ Electret DEGs use as a voltage source an electret which polarizes the dielectric.

It was the aim of this work to develop novel silicone based elastomers with increased permittivity and to evaluate their potential as dielectric to harvest energy from mechanical motion using an electret DEG setup. To achieve this, a nanospring carbon nanotubes filler (NSCNTs) recently developed at KIST was used as filler to increase the dielectric permittivity of either a PDMS or a poly(ethylthiopropyl

This document is the accepted manuscript version of the following article:

Yun Jae Lee, Philip Caspari, Dorina M. Opris, Frank A. Nüesch, Sora Ham, Jung-Hyuk Kim, Sung-Ryong Kim, Byeong-Kwon Ju, and Won Kook Choi, Electrical Energy Generated by Silicone Elastomers Filled with Nanospring-Carbon-Nanotubes, 2019, Journal of Materials Chemistry C. 
methyl)siloxane matrix. The composites were cross-linked with polar triethoxysilane cross-linkers via a condensation reaction catalyzed by tin. Composites containing 10 wt\% NS-CNTs filler were prepared and the influence of the filler on the mechanical properties of the silicone composites was investigated by tensile tests and dynamic-mechanical analysis (DMA). The homogeneity of the filler dispersion in the composites was proven by scanning electron microscopy. The dielectric properties were evaluated by impedance spectroscopy. Finally, the performance of the prepared materials as dielectric in electret DEGs was also evaluated.

\section{Results and discussion}

Scheme 1 shows an overview of all materials synthesized. A silanol terminated polydimethylsiloxane and a poly(methyl propylthioethyl)siloxane P3, prepared in our lab, were cross-linked with either 2-cyano-triethoxysilane or 3-chloropropyltriethoxysilane, respectively via a condensation reaction catalyzed by dibutyltin dilaurate to form materials PDMS-CN and P3-Cl. ${ }^{51}$ For both matrices, an excess of triethoxysilane reagents was used. As a result, both matrices contain silsesquioxane structures, which are generated in-situ during cross-linking. ${ }^{52}$ To increase the dielectric permittivity of PDMS-CN and P3-Cl matrices, NS-CNTs filler was used. This filler is known and was prepared according to the literature. ${ }^{53}$ Shortly, its synthesis started from functionalized single-wall CNTs and zinc acetate at elevated temperatures, where ZnO nanoparticles coated with NS-CNTs are formed. ${ }^{53}$ The formed particles are thereafter treated with $\mathrm{HCl}$ to release the NS-CNTs. The formed NS-CNTs filler has a nanometer size and has been successfully used to enhance the dielectric permittivity of poly(vinylidenefluoride-co-trifluoroethylene). ${ }^{53}$ It is well dispersible in dimethylformamide (see SI), however it cannot be dispersed in common solvents used for processing polysiloxanes, like tetrahydrofuran. Therefore, the first challenge to overcome was to increase the compatibility of the filler with the silicone matrices. This was achieved by a surface treatment of NS-CNTs with 2cyanoethyl(triethoxy)silane as described in the experimental part. For the composites, $10 \mathrm{wt} \%$ of NS-CNTs filler was used in either PDMSCN or P3-Cl with formation of materials PDMS-CN-CNT and P3-CI-CNT. The homogeneity of the filler dispersion in the composites was proven by scanning electron microscopy (Fig. S2). The particles are evenly distributed in the matrix and no agglomerates can be observed.

As reference materials the commercial silicone Elastosi ${ }^{\oplus}$ Film and PDMS prepared from a hydroxyl terminated polydimethylsiloxane cross-linked with poly(methylhydrosiloxane-co-dimethylsiloxane) cross-linker with a thickness of $200 \mu \mathrm{m}$ were used. The impact of NSCNTs filler on different materials properties was evaluated and the obtained results were compared with the reference materials, PDMS$\mathrm{CN}$, and $\mathrm{P3}-\mathrm{Cl}$. 
a)

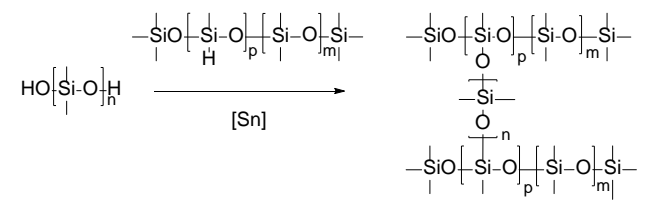

b)

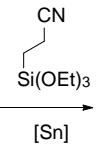

c)

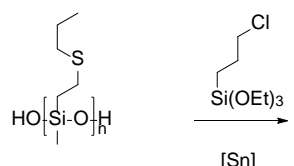

[Sn]

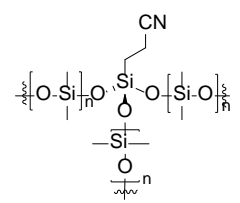

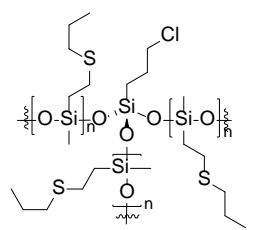
$\mathrm{CN}$<smiles>CCOCCC#N</smiles>

d)

$10 \mathrm{wt} \%$ NS-CNTs

[Sn]

e)<smiles>CCCSCCS(=O)(=O)O</smiles>

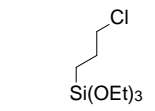

10 wt $\%$ NS-CNTs

[Sn]

PDMS

PDMS-CN

P3-Cl

PDMS-CN-CNT

P3-Cl-CNT

Scheme 1. Overview of all synthesized materials using dibutyltin dilaurate catalyst and: a silanol terminated PDMS matrix and poly(methylhydrosiloxane-co-dimethylsiloxane) cross-liker (a), a silanol terminated PDMS matrix and (2-cyanoethyl)triethoxysilane crosslinker (b), a silanol terminated poly(methylpropylthioethyl)-siloxane (P3) and (3-chloropropyl)triethoxysilane cross-linker (c), a silanol terminated PDMS matrix, (2-cyanoethyl)triethoxysilane cross-linker and 10 wt\% functionalized NS-CNTs (d) and a P3, (3chloropropyl)triethoxysilane cross-linker and $10 \mathrm{wt} \%$ functionalized NS-CNTs (e).

The mechanical properties of the films were evaluated in tensile tests (Fig. 1). The stress-strain curves were averaged from three independent measurements (Fig. S3-S8). The PDMS reference material had an average strain at break of 372\% and an elastic modulus $Y_{10 \%}$ $=0.6 \mathrm{MPa}$, whereas Elastosil ${ }^{\oplus}$ Film had an average strain at break of $485 \%$ and an elastic modulus $Y_{10 \%}=1.2 \mathrm{MPa}$. For PDMS-CN the $Y_{10 \%}$ was about 1.28 MPa and for PDMS-CN-CNT the $Y_{10 \%}$ was slightly lower of about $1.0 \mathrm{MPa}$, due to the lower amount of 2-cyanoethyl(triethoxy)silane used in PDMS-CN-CNT. The average strain at break was 213\% for PDMS-CN and 270\% for PDMS-CN-CNT. The materials prepared starting from $\mathbf{P 3}$ showed rather low strain at break, e.g., P3-Cl and P3-Cl-CNT had an average strain at break of $114 \%$ and of $95 \%$, and an $Y_{10 \%}$ of $0.2 \mathrm{MPa}$ and $0.3 \mathrm{MPa}$, respectively. 


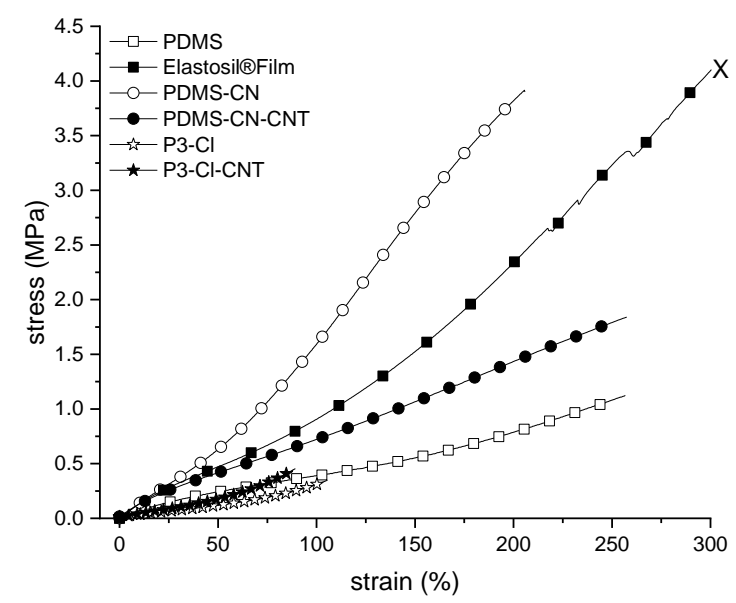

Fig. 1 Stress-strain curves of all materials. They are the average from three independent measurements. The strain at break is the the minimum value obtained from the three tests. In text the avarage of the strain at break from three independent measurements is given.

Dynamic mechanical analysis (DMA) was used to characterize the viscoelastic behavior of all materials (Fig. 2). All thin film elastomers were $2 \%$ strained and tested at a frequency range of $0.05 \mathrm{~Hz}$ to $2 \mathrm{~Hz}$ at $25^{\circ} \mathrm{C}$. We consider $1-2 \mathrm{~Hz}$ as realistic frequency range for DEG applications, i.e. wave energy and human motion harvesters. PDMS and $\mathbf{P 3}-\mathrm{Cl}$ showed the lowest mechanical loss factor tan $(\delta)$ which was around 0.01 . The loss factor $\tan (\delta)$ of Elastosil ${ }^{\circledR}$ Film, PDMS-CN, PDMS-CN-CNT was also very low around 0.03. P3-Cl-CNT showed a considerable increase of mechanical losses $\tan (\delta)$ to about 0.08 at $1 \mathrm{~Hz}$, which may be an indication of a sluggish cross-linking reaction of P3 in presence of NS-CNTs filler.

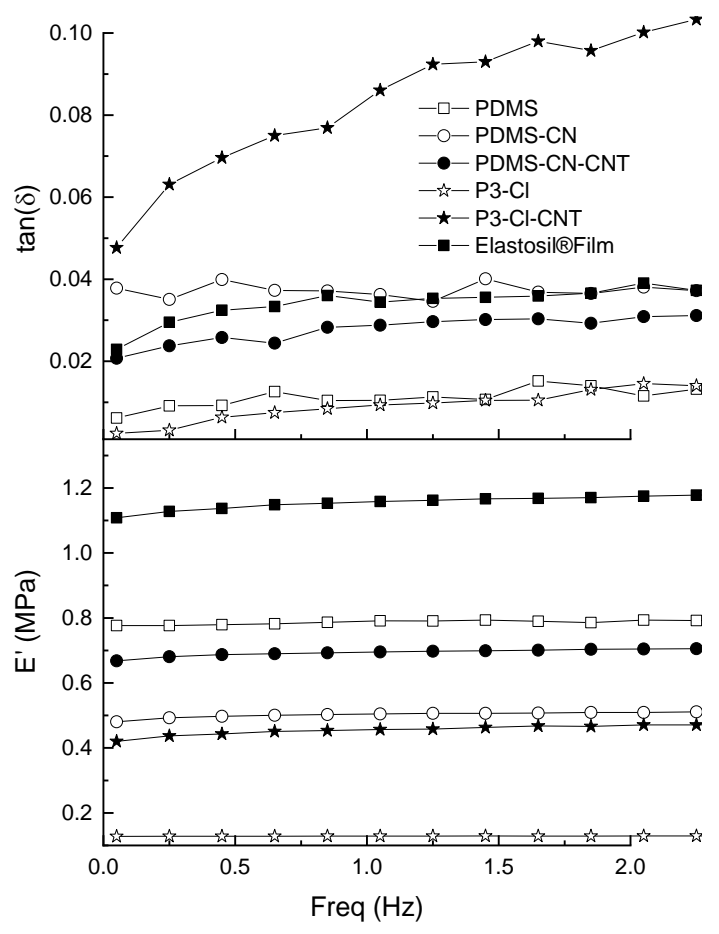

Fig. 2 Dynamic mechanical analysis of all materials at $2 \%$ strain and at a frequency range between 0.05 to $2 \mathrm{~Hz}$. 


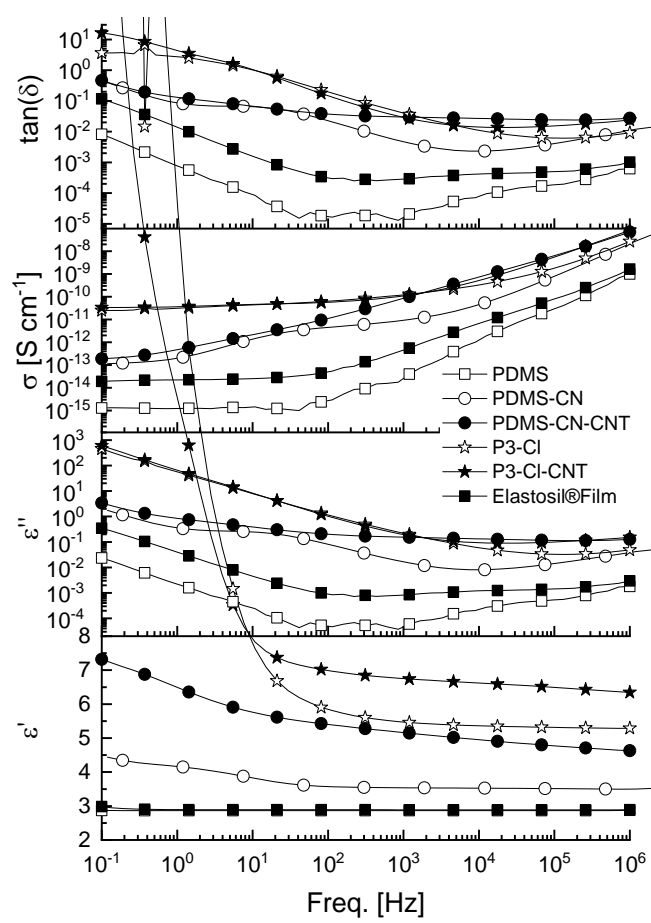

Fig. 3 Dielectric properties of all materials in the frequency range of $10^{-1}$ to $10^{6} \mathrm{~Hz}$.

The dielectric properties were measured in a frequency range from $10^{-1} \mathrm{~Hz}$ to $10^{6} \mathrm{~Hz}$ at ambient temperature (Fig. 3, Table 1). PDMS and Elastosil ${ }^{\circledR}$ Film had a permittivity $\varepsilon^{\prime}$ of 2.9 , while PDMS-CN had a permittivity $\varepsilon^{\prime}$ of 3.5 , which is similar to the value previously reported. ${ }^{51,54}$ The permittivity of the composites at frequencies above $10^{3} \mathrm{~Hz}$ was constant and reached values of 4.6, 5.3, and 6.3 for PDMS-CN-CNT, P3$\mathbf{C l}$, and $\mathbf{P 3}-\mathbf{C l}-\mathbf{C N T}$, respectively. A strong increase in $\varepsilon^{\prime}$ for $\mathbf{P 3}-\mathbf{C l}$ and $\mathbf{P 3}-\mathbf{C l}-\mathbf{C N T}$ at low frequencies was observed, which is caused by electrode polarization due to ionic conductivity. ${ }^{55}$ Contrary to CNT/PDMS composites previously reported where a rather low amount of filler is needed to reach the percolation threshold, our composites showed excellent dielectric properties at $10 \mathrm{wt} \%$ filler. ${ }^{44}$ The reason for this is the nano size of the NS-CNTs filler used and the increased compatibility of the filler with the matrices used which prevents agglomeration. The highest conductivity of $2.5 \times 10^{-11} \mathrm{~S} \mathrm{~cm}^{-1}$ and $3.4 \times 10^{-11} \mathrm{~S} \mathrm{~cm}^{-1}$ was measured for P3-Cl and P3-Cl-CNT, respectively, while all the other materials showed a conductivity which was below $2 \times 10^{-13} \mathrm{~S} \mathrm{~cm}^{-1}$. For materials PDMS-CN and PDMS-CN-CNT the conductivity was $1.2 \times 10^{-13} \mathrm{~S} \mathrm{~cm}^{-1}$ and $1.9 \times 10^{-13} \mathrm{~S} \mathrm{~cm}^{-1}$. The lowest conductivity was measured for the silicone films. The conductivity of PDMS was $1.3 \times 10^{-15} \mathrm{~S} \mathrm{~cm}^{-1}$ and of Elastosil ${ }^{\oplus}$ Film was $1.9 \times 10^{-14} \mathrm{~S} \mathrm{~cm}^{-1}$.

The dielectric breakdown experiments were conducted by placing the dielectric films between two rigid electrodes of $0.25 \mathrm{~mm}^{2}$ area. ${ }^{45}$ For each material ten samples were measured and the average is given in Table 1 . The highest dielectric breakdown above $70 \mathrm{~V} / \mu \mathrm{m}$ was measured for the pure silicone films. The dielectric breakdown decreased for the materials modified with polar groups. For example, the dielectric breakdown was $52 \mathrm{~V} / \mu \mathrm{m}$ for $\mathbf{P 3}-\mathrm{Cl}$ and $59 \mathrm{~V} / \mu \mathrm{m}$ for PDMS-CN, respectively. The incorporation of NS-CNTs resulted in a further reduction of the dielectric breakdown to $48 \mathrm{~V} / \mu \mathrm{m}$ for P3-Cl-CNT and $41 \mathrm{~V} / \mu \mathrm{m}$ for PDMS-CN-CNT, respectively. The dielectric breakdown field is a critical material parameter for regular DEG, where the amount of energy is increasing with the applied voltage. For the electret DEG the dielectric breakdown is less critical, since the electric fields involved in these devices are far below the dielectric breakdown field. Additionally, the dielectric exposure time to maximum electric field is rather short, contrary to DEG where the dielectric is subjected for longer periods of time to rather strong electric fields. Therefore the probability of dielectric failure in electret DEG is significantly lower as compared to regular DEG.

Table 1. Dielectric properties of different materials investigated.

\begin{tabular}{|c|c|c|c|c|c|}
\hline Entry & $\varepsilon^{\prime a}$ & $\varepsilon^{\prime \prime a}$ & $\tan (\delta)^{\mathrm{a}}$ & $\sigma\left[\mathrm{S} \mathrm{cm}^{-1}\right]^{\mathrm{b}}$ & $E_{\max }{ }^{c}[\mathrm{~V} / \mu \mathrm{m}]$ \\
\hline PDMS & 2.9 & $2 \times 10^{-3}$ & $6 \times 10^{-4}$ & $1.3 \times 10^{-15}$ & $>71$ \\
\hline Elastosil ${ }^{\oplus}$ Film & 2.9 & $3 \times 10^{-3}$ & $1 \times 10^{-3}$ & $1.9 \times 10^{-14}$ & $82 \pm 6^{d}$ \\
\hline PDMS-CN & 3.5 & $4 \times 10^{-2}$ & $1 \times 10^{-2}$ & $1.2 \times 10^{-13}$ & $59 \pm 8$ \\
\hline PDMS-CN-CNT & 4.6 & $1 \times 10^{-1}$ & $3 \times 10^{-2}$ & $1.9 \times 10^{-13}$ & $41 \pm 5$ \\
\hline P3-Cl & 5.3 & $5 \times 10^{-2}$ & $9 \times 10^{-3}$ & $2.5 \times 10^{-11}$ & $52 \pm 6$ \\
\hline P3-Cl-CNT & 6.3 & $2 \times 10^{-2}$ & $2 \times 10^{-2}$ & $3.4 \times 10^{-11}$ & $48 \pm 10$ \\
\hline
\end{tabular}




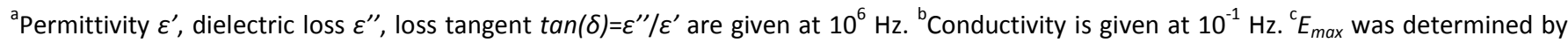
placing the dielectric film between rigid electrodes of $0.25 \mathrm{~mm}^{2}$ area. The average of ten measurements is given. ${ }^{\mathrm{d}}$ Average film thickness of $100 \mu \mathrm{m}$.

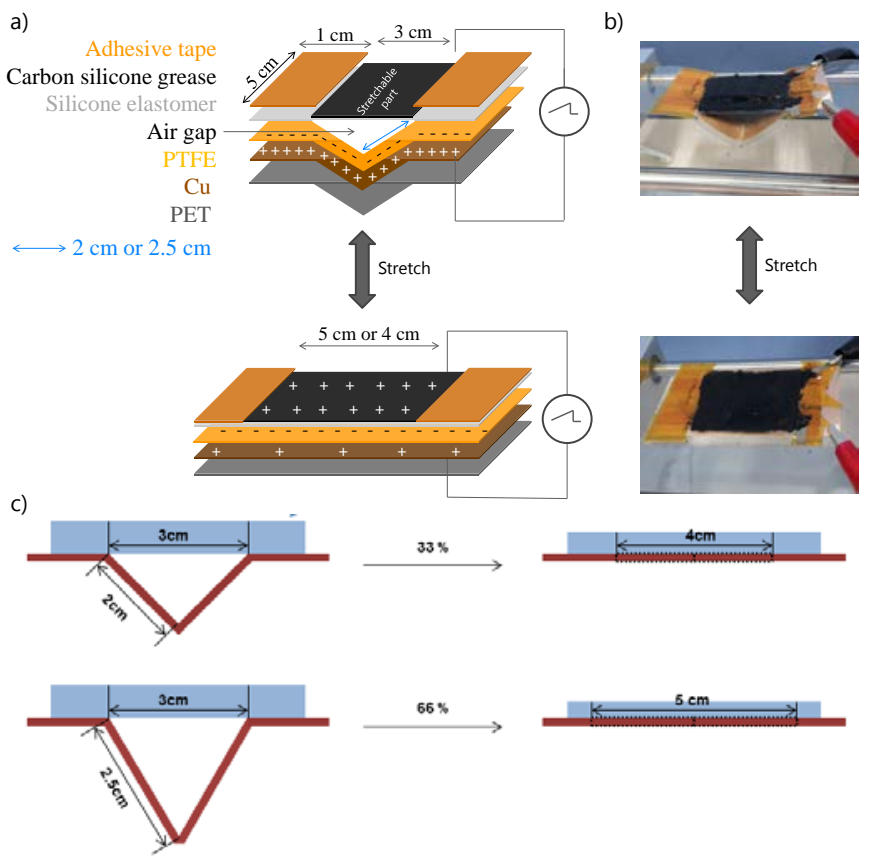

Fig. 4 Construction and working principle of the electret DEG (a). Photos of the custom-made electret DEG in the relaxed (top) or stretched state (bottom) (b). Simplified representation of how the air gap is changing when the electret is stretched by $33 \%$ (from $3 \mathrm{~cm}$ to $4 \mathrm{~cm}$ ) (top) or by $66 \%$ (from $3 \mathrm{~cm}$ to $5 \mathrm{~cm}$ ) (right). The rigid frame unfolded during stretching and folded back during relaxing of the DE.

Finally, experiments to evaluate the potential of all materials as dielectric to harvest energy from mechanical motion were conducted using a home-made electret DEG test device similar to that reported by Jean-Mistral et al., ${ }^{50}$ but with some simplifications (Fig. 4 and Video in the Supporting Information). We used an electret as a voltage source consisting of a Teflon film charged by corona discharge at an initial surface potential of $1000 \mathrm{~V}$. Because most electret materials reported to date are rigid, they cannot be reversibly stretched. To be nevertheless able to stretch them, the electret was shaped in an accordion like structure (Fig. 4). ${ }^{48,50}$ The electret DEG consisted of both rigid and elastic materials. The rigid part, on which one electrode and the charged electret were fixed, had an accordion like structure which allowed unfolding and expansion. The elastic film, on top of which the second stretchable electrode was applied, was fixed above the accordion structure. In the relaxed form, a large air gap between the electret and elastomer exists. Therefore almost all compensation charge is located on the bottom electrode close to the electret (Fig. 4a top). When the device is stretched, the accordion like structure deforms. This deformation is followed by the elastomeric part which is elongated. The air gap distance between electret and elastomer is significantly decreased, and in an ideal case eliminated (Figure 4a bottom). The electret polarizes the dielectric elastomer and charges appear on the top electrode. At the same time the capacitance of the dielectric elastomer changes. In the relaxed state, the capacitance of electret DEG is dominated by the Teflon electret. In the strained state, the capacitance of the DE film exceeds the one of Teflon and reaches:

$C=\varepsilon^{\prime} \varepsilon_{0}^{\prime} A / d^{\prime}$

where $\varepsilon^{\prime}$ is the permittivity of the elastomer, $\varepsilon_{0}^{\prime}$ is the permittivity of air, $A$ is the surface area of the flexible electrode and $d$ is the thickness of the dielectric elastomer. Re-arrangement of charges between the two electrodes through a load due to changes in capacitance during strain-relaxation cycles generates an alternative current. The capacitance of the electret DEG is inversely proportional to the dielectric thickness. ${ }^{48}$ A higher DEG output voltage is expected for thinner films. Because, materials P3-Cl and P3-Cl-CNT showed a rather low strain at break of $100 \%$ only, thin films constructed from them were difficult to handle and to use in electret DEG construction. To be nevertheless able to compare the properties of different materials, films with a thickness of $200 \mu \mathrm{m}$ were tested. These films were easier to handle and showed good mechanical stability in devices, except P3-CI-CNT which ruptured easily.

The active part of the dielectric elastomer of the device has an area of $3 \mathrm{~cm} \times 5 \mathrm{~cm}$. The total weight of the electret DEG was merely $8 \mathrm{~g}$. As DEGs are studied with regard to wearable electronics, the device weight is considered an essential aspect (video in Supporting Information). The rigid frame unfolded during stretching and folded back during relaxing of the DE and defined the maximum elongation of the active part of the dielectric elastomer (Fig. 4). The maximum amount of strain applied to the dielectric elastomer is given by the length of the flexible (not elastic) electret, which is $4 \mathrm{~cm}$ and $5 \mathrm{~cm}$ for the generators strained by $33 \%$ and by $66 \%$, respectively. The high sensitivity of the DEG's output voltage against the remaining air gap between electret and DE was the main experimental challenge for 
electret DEG measurements. Only when the electret unfolded completely, the output voltage was maximized. To minimize this source of uncertainty, five generators were tested for each material at each strain, except for PDMS-CN generator stretched by $33 \%$, where we ran out of material and measured only four generators. All generator measurements are included in the supporting information (Fig. S9-S17. Not all generators performed equally well, mainly due to the difficulty of completely removing any air gap in the stretched state. We consider, however, that the best performant generator illustrates material performance and not the artifacts from electret DEG sample construction/operation.

The voltage generated by different materials at two different strains of $33 \%$ and $66 \%$ and at a frequency of $1 \mathrm{~Hz}$ was measured using a Tektronix 'TDS 2024B' oscilloscope with an input impedance $1 \mathrm{M} \Omega$ in parallel with a capacitance of 20 pF. No external resistor was used at the entrance.

A typical response is shown in Fig. 5 for a DEG constructed with PDMS-CN-CNT at $66 \%$ strain. Table 2 summarizes the maximum output voltage (best performant generator) and the average output voltage of different materials tested in DEG at two different strains, as well as the permittivity $\varepsilon^{\prime}$ and the maximum tensile strain of the DE. The voltage peaks occurred only when the dielectric membrane was in close contact to the electret during stretching and relaxation. It was noted that the voltage signal registered during stretching and relaxation was in many cases not symmetrical, and the signal measured during relaxation was smaller and wider. We attribute this feature to the adhesion of the dielectric elastomer on the electret layer which slows down the separation process when the DEG is relaxing.

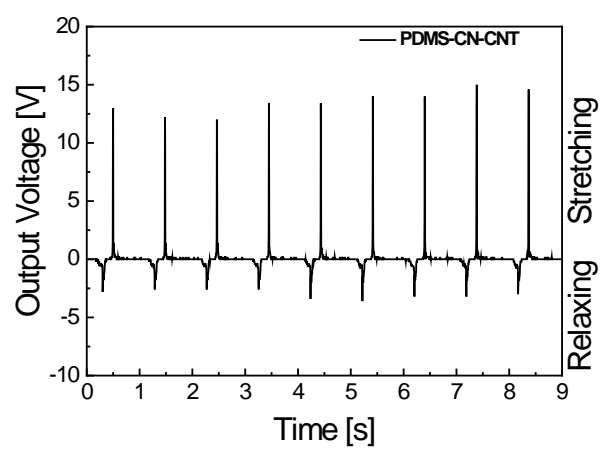

Fig. 5 The output voltage versus time of a DEG constructed with PDMS-CN-CNT as dielectric elastomer which was strained $66 \%$ at $1 \mathrm{~Hz}$.

The full potential of the materials using our electret setup can hardly be evaluated, since it does not allow exploring the full range of strains. The theoretical electrical energy $\Delta E$ released during one stretching transformation can be calculated according to (see $\mathrm{SI}$ ):

$$
\Delta E=\frac{1}{2} V_{0}^{2} \cdot \frac{C_{1} C_{2}}{C_{1}+C_{2}}
$$

where $V_{0}=1000 \mathrm{~V}$ is the surface potential induced by the initial discharge on the Teflon electret, $C_{2}$ and $C_{1}$ is the capacitance of the electret and stretched DE film, respectively. Note that the reduction in film thickness upon straining was taken into account in the calculation of $\mathrm{C}_{1}$. Taking the maximum strain values in Figure 1 and the relative permittivities in Table 2, we obtain increasing values $\Delta E$ of $3.13 \cdot 10^{-4} \mathrm{~J}, 3.41 \cdot 10^{-4} \mathrm{~J}, 3.86 \cdot 10^{-4} \mathrm{~J}$ and $4.95 \cdot 10^{-4} \mathrm{~J}$ for PDMS-CN-CNT, PDMS-CN, PDMS, and Elastosil, respectively. If the strain is fixed to $66 \%$, we obtain decreasing values $\Delta E$ of $1.1910^{-4} \mathrm{~J}, 1.1010^{-4} \mathrm{~J}$, and $1.0210^{-4} \mathrm{~J}$ for PDMS-CN-CNT, PDMS-CN, and PDMS, respectively. Thus the theoretical amount of released electrical energy at a predetermined strain is higher for PDMS-CN-CNT.

Table 2. Output voltage of the best performant DEG as well as the average of five DEG at $33 \%$ and $66 \%$ lateral strain, the dielectric permittivity $\varepsilon^{\prime}$, the maximum tensile strain, and the calculated energy at $33 \%, 66 \%$ strain, and at maximum strain $s_{\max }$ respectively.

\begin{tabular}{lccccccccc}
\hline Entry & $\begin{array}{c}\mathrm{V}_{\max } \\
\text { at } 33 \%^{\mathrm{a}} \\
{[\mathrm{V}]}\end{array}$ & $\begin{array}{c}\mathrm{V}_{\max } \\
\text { at } 66 \%^{\mathrm{b}} \\
{[\mathrm{V}]}\end{array}$ & $\begin{array}{c}\mathrm{V}_{\text {average }} \\
\text { at } 33 \%{ }^{\mathrm{c}} \\
{[\mathrm{V}]}\end{array}$ & $\begin{array}{c}\mathrm{V}_{\text {average }} \\
\text { at } 66 \%^{\mathrm{d}} \\
{[\mathrm{V}]}\end{array}$ & $\mathcal{E}^{\mathrm{e}}$ & $\begin{array}{c}s_{\max } \\
{[\%]}\end{array}$ & $\begin{array}{c}\Delta E_{33 \%^{\mathrm{f}}} \\
{[\mathrm{J}]}\end{array}$ & $\begin{array}{c}\Delta E_{66 \%}{ }^{\mathrm{f}} \\
{[\mathrm{J}]}\end{array}$ & $\begin{array}{c}\Delta E_{\max } \\
{[\mathrm{J}]}\end{array}$ \\
\hline PDMS & $5.1 \pm 0.2$ & $9.3 \pm 0.2$ & $3 \pm 1$ & $5 \pm 1$ & $2.9 \pm 0.15$ & 372 & $-7.4810^{-5}$ & $-1.0210^{-4}$ & $-3.8610^{-4}$ \\
Elastosil ${ }^{-5}$ Film & $4.5 \pm 0.1$ & $8.6 \pm 0.1$ & $3 \pm 1$ & $7 \pm 1$ & $2.9 \pm 0.15$ & 485 & $-7.4810^{-5}$ & $-1.0210^{-4}$ & $-4.9510^{-4}$ \\
PDMS-CN & $5.1 \pm 0.2$ & $6.8 \pm 0.2$ & $4 \pm 1$ & $7 \pm 1$ & $3.5 \pm 0.20$ & 313 & $-8.0810^{-5}$ & $-1.1010^{-4}$ & $-3.4110^{-4}$ \\
PDMS-CN-CNT & $8.8 \pm 0.3$ & $14.5 \pm 0.3$ & $5 \pm 1$ & $11 \pm 2$ & $4.6 \pm 0.25$ & 270 & $-8.9210^{-5}$ & $-1.1910^{-4}$ & $-3.1310^{-4}$ \\
P3-Cl & $8.1 \pm 1$ & - & $5 \pm 1$ & - & $5.4 \pm 0.25$ & 114 & $-9.3210^{-5}$ & $-1.2410^{-4}$ & $-1.6910^{-4}$ \\
P3-Cl-CNT & - & - & - & - & $6.3 \pm 0.3$ & 95 & $-9.7910^{-5}$ & $-1.2910^{-4}$ & $-1.5610^{-4}$ \\
\hline
\end{tabular}

The voltage of the best performant generator at $1 \mathrm{~Hz}$ which is the average of nine cyclic measurements including corresponding standard deviation at a strain of ${ }^{a} 33 \%$ and ${ }^{b} 66 \%$, respectively. The average voltage of five generators at ${ }^{c} 33 \%$ and at ${ }^{d} 66 \%$ strain, respectively. ${ }^{\mathrm{e}}$ Permittivity value taken at $10^{6} \mathrm{~Hz}$. ${ }^{\mathrm{f}}$ Calculations of $\Delta E$ at $33 \%$ and $66 \%$ strain as well as at $s_{\max }$ can be found in the SI. 


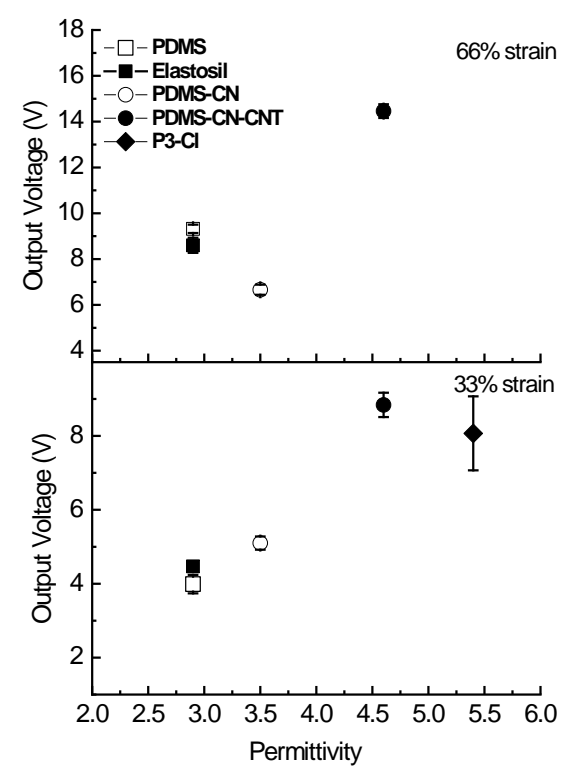

Fig. 6 Output voltage of the best performant DEG versus permittivity $\varepsilon^{\prime}$ of the DE at $33 \%$ and at $66 \%$ strain.

Fig. 6 shows the output voltage of different materials as function of permittivity for $33 \%$ and $66 \%$ strain. Material P3-Cl-CNT has the highest permittivity of $\varepsilon^{\prime}=6.3$ and thus should perform best in generator. However, it ruptured easily and therefore no DEGs could be constructed with this material. P3-Cl ruptured when $66 \%$ strain was used and therefore the harvested voltage could be measured only at $33 \%$ strain. Material PDMS-CN-CNT generated the highest average output voltage of $8.8 \mathrm{~V}$ and $14.5 \mathrm{~V}$ at $33 \%$ and $66 \%$ strain, respectively which is almost two times higher as compared to Elastosil ${ }^{\circledR}$ Film with $4.5 \mathrm{~V}$ and $8.6 \mathrm{~V}$ at the same strains. Most of the materials showed the expected trend, e.g., an increase in the generated voltage with increasing the permittivity except for material $\mathbf{P 3}-\mathbf{C l}$ at $33 \%$ strain and PDMS-CN at $66 \%$ strain. This inconsistency may be explained by the somewhat higher dielectric losses of these materials or due to the air gap which may vary slightly for different measurements. While the dielectric permittivity strongly influences the voltage output, other materials properties such as mechanical stability, e.g. the maximum achievable strain at break have a strong impact on generator performance as well. ${ }^{56}$ The generated voltage can be increased by using materials with even higher dielectric permittivity, by reducing the thickness of the film, and by using larger stains while the air gap between the electret and the stretched elastomer should be minimized. A direct comparison of the performance of the materials developed in this work and other materials from the literature tested in DEG cannot be done easily because of the different setup used in different laboratories. ${ }^{57}$ It should be mention that in electret DEGs only regular silicones were investigated and we compared the performance of our materials with Elastosil ${ }^{\circledR}$ Film. The higher generated voltage for the composite containing NS-CNTs as compared to Elastosil ${ }^{\otimes}$ Film, clearly show the positive effect of the filler used.

Conventional DEGs require operation near dielectric breakdown and have rather complicated charge/discharge cycle. Contrary to $D E G s$, electret DEGs are very unlikely to suffer a breakdown and are thus more reliable. While DEGs may be superior to electret DEGs in terms of energy harvested per cycle, the safer operation of electret DEGs over the conventional DEGs allows electret DEGs to be operated on humans (see Movie in Supporting Information). Therefore, applications of electret DEGs to recharge portable devices can be envisioned. ${ }^{58}$

\section{Experimental}

Unless otherwise stated, all chemicals were reagent grade and used without purification. 1,3,5,7-Tetramethyl-1,3,5,7-tetravinylcyclotetrasiloxane $\left(\mathrm{V}_{4}\right)$, (25-35\% methylhydro-siloxane)dimethyl-siloxane (AB109380) and polydimethylsiloxane, hydroxyl terminated (AB116665, $M_{n}=90000 \mathrm{~g} \mathrm{~mol}^{-1}, M_{\mathrm{w}}=130000 \mathrm{~g} \mathrm{~mol}^{-1}$ ) were purchased from ABCR. Tetrahydrofuran (THF) and dimethylformamide (DMF) were purchased from VWR. THF was stored over sodium under nitrogen atmosphere and distilled before use. Single-walled carbon nanotubes were purchased from Carbon Nanotech, Co., South Korea. (CNTs SP95, >95 wt\%). All other chemicals were purchased from Sigma Aldrich. Poly(ethylthiopropyl methyl)siloxane P3 $\left(M_{n}=90.000 \mathrm{~g} / \mathrm{mol}, M_{w}=185.000 \mathrm{~g} / \mathrm{mol}, P D I=2.0\right)$ was synthesized according to

This document is the accepted manuscript version of the following article:

Yun Jae Lee, Philip Caspari, Dorina M. Opris, Frank A. Nüesch, Sora Ham, Jung-Hyuk Kim, Sung-Ryong Kim, Byeong-Kwon Ju, and Won Kook Choi, Electrical Energy Generated by Silicone Elastomers Filled with Nanospring-Carbon-Nanotubes, 2019, Journal of Materials Chemistry C. 
literature. ${ }^{51}$ Thin films of Elastosil ${ }^{\oplus}$ Film $(200 \mu \mathrm{m}, 100 \mu \mathrm{m}, 50 \mu \mathrm{m}, 20 \mu \mathrm{m})$ were provided from DRAWIN Vertriebs-GmbH, Riemerling while carbon black silicone grease were provided from M.G. Chemicals Ltd.

The tensile tests were performed on a Zwick $Z 010$ tensile test machine with a crosshead speed of $50 \mathrm{~mm} \mathrm{~min}^{-1}\left(278 \% \mathrm{~min}^{-1}\right)$. Dynamic mechanical analysis was carried out on a RSA 3 DMA from TA Instruments. Stripes of $10 \mathrm{~mm} \times 20 \mathrm{~mm}$ were measured applying $2 \%$ strain in the frequency range of $0.05-2 \mathrm{~Hz}$ at $25^{\circ} \mathrm{C}$. $\operatorname{Tan}(\delta)_{D M A}$ is given as the fraction of the imaginary $E^{\prime \prime}$ and real elastic moduli $E^{\prime}$ at $2 \%$ strain. The microstructures of the films were measured with a SEM (FEI Inspect F) on samples prepared by freeze-breaking in liquid nitrogen and sputtered with a thin layer of Pt. Permittivity measurements were performed in the frequency range from $10^{-1} \mathrm{~Hz}$ to $10^{6} \mathrm{~Hz}$ using a Novocontrol Alpha-A frequency analyzer. The $V_{R M S}$ (root-mean-square voltage) of the probing AC electric signal applied to the samples was $1 \mathrm{~V}$. Au electrodes with a thickness of $20 \mathrm{~nm}$ were sputtered on both sides of the film. The permittivity $\varepsilon^{\prime}$ was determined from the capacitance $C=\varepsilon^{\prime} \varepsilon_{0} A / d$, where $A$ is the electrode area, $d$ is the thickness of the film, and $\varepsilon_{0}$ is the vacuum permittivity. The thickness of the film was measured by a micrometer gauge with an uncertainty of $\pm 5 \mu \mathrm{m}$. Two stainless steel discs with a diameter of $20 \mathrm{~mm}$ served as electrodes. The samples were annealed at $80^{\circ} \mathrm{C}$ for $18 \mathrm{~h}$ at $10 \mathrm{mbar}$ to remove any residual solvents.

For the generator, a poly(ethylene terephthalate) (PET) film $(130 \mu \mathrm{m})$ was used as substrate on which a copper tape (75 $\mu \mathrm{m})$ was attached, which served as bottom electrode. On the bottom electrode, the poly(tetrafluoroethylene) (PTFE) electret film (140 $\mu \mathrm{m})$ was attached. The electret was prepared using a high frequency corona discharge generator from Electro-Technic Products Inc. (Model BD10AS) with an output of $20 \mathrm{kV}$ to $45 \mathrm{kV}$ at a frequency of approximately $500 \mathrm{kHz}$. An electrostatic voltmeter (Model 347, TREK, INC) was used to measure the induced surface potential of the electret. PTFE surface potential was $1000 \mathrm{~V} \pm 100 \mathrm{~V}$. On top of PET/Cu/PTFE layers (rigid part), which was bended in an accordion like shape, the dielectric film $(5 \mathrm{~cm} \times 5 \mathrm{~cm})$ coated with carbon black silicone grease top electrode $(3 \mathrm{~cm} \times 5 \mathrm{~cm})$ was attached on the passive part of the dielectric using an adhesive tape. A large air gap between the dielectric and the electret formed (Fig. 4). The rigid part unfolded during stretching the elastomer and folded back due to the elastic restoring forces in the dielectric elastomer. The total length of the DEG device in the stretched form was $20 \mathrm{~cm} \times 6 \mathrm{~cm}$. The DEG measurement setup was equipped with a mechanical stretcher and an electrical motor (see Supporting Information). The DEGs were stretched by $33 \%$ (from $3 \mathrm{~cm}$ to $4 \mathrm{~cm}$ ) or by $66 \%$ (from $3 \mathrm{~cm}$ to $5 \mathrm{~cm}$ ). The output voltage was measured using Tektronix 'TDS 2024B' oscilloscope with an input impedance of $1 \mathrm{M} \Omega$ in parallel with a capacitance of $20 \mathrm{pF}$. No external resistor was used at the entrance.

General synthesis of the silicone elastomer thin films

A solution of hydroxyl terminated PDMS or P3, cross-linker, and catalytic amounts of dibutyltin dilaurate in THF was stirred and sonicated for 3 minutes to remove the air bubbles (see Table 3). Thin films were made on a glass coated with PTFE or on a PET substrate by doctor blade technique. The films were stored in a closed chamber at ambient temperature of $25^{\circ} \mathrm{C}$ and humidity of $30 \%$ for $48 \mathrm{~h}$. They were then dried at $80^{\circ} \mathrm{C}$ in vacuo for $12 \mathrm{~h}$, left at room temperature for at least three weeks, before further testing.

Table 3. Amount and type of reagents used in the synthesis of different materials.

\begin{tabular}{llll}
\hline Entry $^{\mathrm{a}}$ & $\begin{array}{l}\text { Matrix } \\
\text { [amount] }\end{array}$ & Cross-linker [amount] & $\begin{array}{l}\text { NS-NTs } \\
\text { [amount] }\end{array}$ \\
\hline PDMS & $\mathrm{AB} 109380[1 \mathrm{~g}]$ & $\mathrm{AB} 109380[0.1 \mathrm{~g}]$ & - \\
PDMS-CN & $\mathrm{AB} 109380[0.6 \mathrm{~g}]$ & $\mathrm{CN}-\mathrm{CL}^{\mathrm{b}}[0.4 \mathrm{~g}]$ & - \\
PDMS-CN-CNT & $\mathrm{AB} 109380[0.57 \mathrm{~g}]$ & $\mathrm{CN}-\mathrm{CL}^{\mathrm{b}}[0.33 \mathrm{~g}]$ & $0.1 \mathrm{~g}$ \\
P3-Cl & $\mathrm{P3}[0.7 \mathrm{~g}]$ & $\mathrm{Cl}^{-C^{c}}[0.175 \mathrm{~g}]$ & - \\
P3-Cl-CNT & P3 $[0.72 \mathrm{~g}]$ & $\mathrm{Cl}^{\mathrm{C}} \mathrm{CL}[0.18 \mathrm{~g}]$ & $0.1 \mathrm{~g}$ \\
\hline
\end{tabular}

${ }^{\mathrm{a}} 1 \mathrm{~g}$ polymer and dibutyltin dilaurate $(40 \mu \mathrm{l})$ in THF $(2 \mathrm{ml}),{ }^{\mathrm{b}}$ 2-cyanoethyl(triethoxy)silane, ${ }^{\mathrm{c}}$ 3-chloropropyltriethoxysilane.

General synthesis of silicone composite thin films

NS-CNTs ( $3 \mathrm{~g}$ ) were suspended in DMF (240 ml) and sonicated for 3 minutes with a tip sonicator at $40 \%$ power. To this suspension, 2cyanoethyl(triethoxy)silane $(6 \mathrm{ml})$ was added followed by the ammonium hydroxide aqueous solution $(25 \mathrm{wt} \%, 6 \mathrm{ml})$. The reaction was stirred for 3 days. The precipitate was filtered, washed with ethanol and dispersed in THF. To this dispersion either hydroxyl terminated PDMS or P3, the cross-linker, and the Sn catalyst were added and mechanically mixed. For the amount of reagents used, see Table 3 . The composites were subjected to three-roll milling for approximately 15 minutes. Thin films were made on PTFE coated glass or on PET substrate by doctor blade techniques and stored in a closed chamber at ambient temperature of $25^{\circ} \mathrm{C}$ and humidity of $30 \%$ for $48 \mathrm{~h}$. They were then dried at $80^{\circ} \mathrm{C}$ in vacuo for $12 \mathrm{~h}$ before further testing.

\section{Conclusions}

Novel silicone composites consisting of NS-CNTs into either a PDMS or a chemically modified polysiloxane matrix were prepared. The chemical treatment of the highly polar NS-CNTs filler with (2-cyanoethyl)triethoxysilane increased its compatibility to the polysiloxane matrices used. The composites were homogenous and have low mechanical losses as proven by DMA measurements. Two composites, P3CI-CNT with a permittivity value of $\varepsilon^{\prime}=6.3$, but low tensile strength and a material PDMS-CN-CNT with a permittivity value of $\varepsilon^{\prime}=4.6$ and high tensile strength were prepared. The developed materials were investigated regarding their ability to generate a voltage in a DEG test 
device using an electret as polarizing source. Despite the attractively high dielectric permittivity of $\varepsilon^{\prime}=6.3$ for P3-Cl-CNT, no generators could be constructed with this material, because the films ruptured. An increase of the voltage generated, from $8.6 \mathrm{~V}$ for Elastosil ${ }^{\circledR}$ Film $\left(\varepsilon^{\prime}\right.$ $=3)$ to $14.5 \mathrm{~V}$ for PDMS-CN-CNT $\left(\varepsilon^{\prime}=4.6\right)$ was observed. While the increase in output voltage is related to the increased permittivity other materials and device related factors may also be influential.

The mechanical stability of PDMS-CN-CNT is not only demonstrated by the tensile tests but also by applying a $66 \%$ strain on the PDMS-CNCNT thin film in the electret DEG operated at $1 \mathrm{~Hz}$ for 9 cycles. The safer and easy operation of electret DEGs allows their use on the humans and may be used one day for recharging portable electronic devices. Future work will be directed towards optimizing the generator setup and evaluating other high permittivity materials in electret DEGs.

\section{Conflicts of interest}

There are no conflicts to declare.

\section{Acknowledgements}

We gratefully acknowledge the financial support from KIST Institutional Research Program (2V04270, 2V04580, and 2V05320), from Empa (International Cooperation Project between KIST and Empa), SNF 200020-172693, as well as to the Korean-Swiss Young Researchers Exchange Program. We also acknowledge B. Fischer and T. Kuenniger (both Empa) for their support with GPC and DMA measurements, respectively.

+ Y.J.Lee and P. Caspari contributed equally to this work.

\section{Notes and references}

1 P. Brochu, W. Yuan, H. Zhang and Q. Pei, ASME Conf. Smart Mater. Adapt. Struct. Intell. Syst. Proc., 2009, 1, $197-204$.

2 R. D. Kornbluh, R. Pelrine, H. Prahlad, A. Wong-Foy, B. McCoy, S. Kim, J. Eckerle and T. Low, MRS Bull., 2012, 37, $246-253$.

3 S. Bauer, S. Bauer-Gogonea, I. Graz, M. Kaltenbrunner, C. Keplinger and R. Schwödiauer, Adv. Mater., 2014, 26, $149-162$.

4 R. Kaltseis, C. Keplinger, R. Baumgartner, M. Kaltenbrunner, T. Li, P. Mächler, R. Schwdiauer, Z. Suo and S. Bauer, Appl. Phys. Lett., 2011, 99, 10-13.

5 R. Pelrine, R. D. Kornbluh, J. Eckerle, P. Jeuck, S. Oh, Q. Pei and S. Stanford, Proc. SPIE, 2001, 4329, 148.

6 G. Moretti, M. Righi, R. Vertechy and M. Fontana, Polym., 2017, 9, 283.

7 G. Yin, Y. Yang, F. Song, C. Renard, Z. M. Dang, C. Y. Shi and D. Wang, ACS Appl. Mater. Interfaces, 2017, 9, 5237.

8 S. J. A. Koh, X. Zhao and Z. Suo, Appl. Phys. Lett., 2009, 94, 262902.

9 G. Neidlein, C. Hentschel, N. Scharmann, C. Langenstein, M. Voss, B. Hagemann (Robert Bosch GmbH), DE 102009053393 A1, $2009 .$.

10 R. Van Kessel (Single Buoy Moorings INC.), WO 013/167577 Al, 2013.

11 S. Shian, J. Huang, S. Zhu and D. R. Clarke, Adv. Mater., 2014, 26, 6617-6621.

12 J. Huang, S. Shian, Z. Suo and D. R. Clarke, Adv. Funct. Mater., 2013, 23, 5056-5061.

13 S. Chiba, M. Waki, R. Kornbluh and R. Pelrine, Proc. SPIE, 2008, 6927, 692715.

14 P. Jean, A. Wattez, G. Ardoise, C. Melis, R. Van Kessel, A. Fourmon, E. Barrabino, J. Heemskerk and J. P. Queau, Proc. SPIE, 2012, 8340, $83400 C$.

15 R. Van Kessel, A. Wattez and P. Bauer, Proc. SPIE, 2014, 9056, 90561D.

16 J. Maas and C. Graf, Smart Mater. Struct., 2012, 21, 064006.

17 R. D. Kornbluh, R. Pelrine, Q. Pei, R. Heydt, S. Stanford, S. Oh and J. Eckerle, Proc. SPIE, 2002, 4698, 254.

18 C. Jean-Mistral, S. Basrour and J. Chaillout, Proc. SPIE, 2008, 6927, 692716-1.

19 T. G. McKay, B. M. O’Brien, E. P. Calius and I. A. Anderson, Appl. Phys. Lett., 2011, 98, 2009.

20 J. Biggs, K. Danielmeier, J. Hitzbleck, J. Krause, T. Kridl, S. Nowak, E. Orselli, X. Quan, D. Schapeler, W. Sutherland and J. Wagner, Angew. Chem. Int. Ed., 2013, 52, 9409.

21 R. Kaltseis, C. Keplinger, S. J. Adrian Koh, R. Baumgartner, Y. F. Goh, W. H. Ng, A. Kogler, A. Tröls, C. C. Foo, Z. Suo and S. Bauer, RSC Adv., 2014, 4, 27905-27913.

22 S. E. Chen, L. Deng, Z. C. He, E. Li and G. Y. Li, Smart Mater. Struct., 2016, 25, 055017.

23 T. McKay, B. O'Brien, E. Calius and I. Anderson, Proc. SPIE, 2012, 8340, 83401Y.

24 T. McKay, B. O’Brien, E. Calius and I. Anderson, Appl. Phys. Lett., 2010, 97, 2008.

25 I. A. Anderson, E. P. Calius, T. Gisby, T. Mckay, I. A. Anderson, E. P. Calius, T. Gisby, T. Hale, T. Mckay, B. O. Brien and S. Walbran, Proc. SPIE, 2009, 7287, 72871 $\mathrm{H}-1$.

26 F. Foerster and H. F. Schlaak, Proc. SPIE, 2014, 9056, 905637.

27 C. Jean-Mistral, T. Vu-Cong and A. Sylvestre, Smart Mater. Struct., 2013, 22, 104017.

28 C. Tugui, C. Ursu, L. Sacarescu, M. Asandulesa, G. Stoian, G. Ababei and M. Cazacu, ACS Sustain. Chem. Eng., 2017, 5, 7851. 
29 Y. Chiu and S. H. Wu, J. Phys. Conf. Ser., 2013, 476, 012037.

30 P. Brochu, H. Stoyanov, R. Chang, X. Niu, W. Hu and Q. Pei, Adv. Energy Mater., 2014, 4, 1-9.

31 C. Jordi, A. Schmidt, G. Kovacs, S. Michel and P. Ermanni, Smart Mater. Struct., 2011, 20, 075003.

32 L. Maffli, S. Rosset, M. Ghilardi, F. Carpi and H. Shea, Adv. Funct. Mater., 2015, 25, 1656.

33 D. M. Opris, Adv. Mater., 2018, 30, 1703678.

34 F. B. Madsen, A. E. Daugaard, S. Hvilsted and A. L. Skov, Macromol. Rapid Commun., 2016, 37, 378.

35 E. Bortot, R. Springhetti and M. Gei, J. Eur. Ceram. Soc., 2014, 34, 2623-2632.

36 G. Boccalero, C. Jean-Mistral, M. Castellano and C. Boragno, Compos. Part B Eng., 2018, 146, 13-19.

37 W. Hu, S. N. Zhang, X. Niu, C. Liu and Q. Pei, J. Mater. Chem. C, 2014, 2, 1658-1666.

38 H. Böse, D. Uhl and R. Rabindranath, Proc. SPIE, 2012, 8340, 83402E.

39 B. Kussmaul, S. Risse, G. Kofod, R. Waché, M. Wegener, D. N. McCarthy, H. Krüger and R. Gerhard, Adv. Funct. Mater., 2011, $21,4589$.

40 C. Racles, M. Alexandru, A. Bele, V. E. Musteata, M. Cazacu and D. M. Opris, RSC Adv., 2014, 4, 37620.

41 S. J. Duenki, Y. S. Ko, F. A. Nueesch and D. M. Opris, Adv. Funct. Mater., 2015, 25, 2467.

42 M. Stepp, F. Achenbach and A. Koellnberger, PCT Int. Appl., WO 2015/121261 A1 20150820, 2015.

43 G. Boccalero, C. Jean-Mistral, M. Castellano and C. Boragno, Compos. Part B Eng., 2018, 146, 13.

44 K. Goswami, A. E. Daugaard, A. L. Skov, RSC Adv. 2015, 5, 12792.

45 J. E. Q. Quinsaat, M. Alexandru, F. A. Nüesch, H. Hofmann, A. Borgschulte, D. M. Opris, J. Mater. Chem. A, 2015, 3, 14675-14685.

46 F. Carpi, G. Gallone, F. Galantini and D. De Rossi, Adv. Funct. Mater., 2008, 18, 235.

47 H. Stoyanov, P. Brochu, X. Niu, E. Della Gaspera and Q. Pei, Appl. Phys. Lett., 2012, 100, 2010.

48 T. Vu-Cong, C. Jean-Mistral and A. Sylvestre, Proc. SPIE, 2013, 8687, 86870H.

49 C. Jean-Mistral, T. Porter, T. Vu-Cong, S. Chesné and A. Sylvestre, IEEE/ASME, 2014, 1430.

50 C. Jean-Mistral, T. Vu Cong and A. Sylvestre, Appl. Phys. Lett., 2012, 101, 162901.

51 P. Caspari, S. J. Dünki, F. Nueesch and D. Opris, J. Mater. Chem. C, 2018, 6, 2043.

52 A. Bele, M. Dascalu, C. Tugui, M. lacob, C. Racles, L. Sacarescu and M. Cazacu, Mater. Des., 2016, 106, 454.

53 Y. J. Lee, S. R. Ham, J. H. Kim, T. H. Yoo, S. R. Kim, Y. T. Lee, D. K. Hwang, B. Angadi, W. S. Seo, B. K. Ju and W. K. Choi, Sci. Rep., 2018, 8, 4851.

54 L. Zhang, D. Wang, P. Hu, J.-W. Zha, F. You, S.-T. Li, Z.-M. Dang, J. Mater. Chem. C, 2015, 3, 4883-4889.

55 F. Kremer, A. Schönhals, Broadband Dielectric Spectroscopy, Springer, 2003.

56 D. De Rossi, F. Carpi, F. Galantini, Adv. Sci. Tech. 2009, 57, 247-256.

57 F. Carpi, I. Anderson, S. Bauer, G. Frediani, G. Gallone, M. Gei, C. Graaf, C. Jean-Mistral, W. Kaa, G. Kofod, M. Kollosche, R. Kornbluh, B. Lassen, M. Matysek, S. Michel, S. Nowak, B. O’Brien, Q. Pei, R. Pelrine, B. Rechenbach, S. Rosset, H. Shea, Smart Mater. Struct. 2015, 24, 105025.

58 S. J. A. Koh, C. Keplinger, T. Li, S. Bauer and Z. Suo, IEEE/ASME Trans. Mechatronics, 2011, 16, 33. 